\section{Invasive Fungal Disease, Isavuconazole Treatment Failure, and Death in Acute Myeloid Leukemia Patients}

\author{
Anne-Pauline Bellanger, Ana Berceanu, \\ Emeline Scherer, Yohan Desbrosses, \\ Etienne Daguindau, Steffi Rocchi, Laurence Millon
}

Author affiliations: University Hospital, Besançon, France

(A.-P. Bellanger, E. Scherer, S. Rocchi, L. Millon); Franche-Comté University, Besançon (A.-P. Bellanger, E. Scherer,

L. Millon); Besançon University Hospital, Besançon (A. Berceanu, Y. Desbrosses, E. Daguindau)

DOI: https://doi.org/10.3201/eid2509.190598

We present 2 fatal cases of invasive fungal disease with isavuconazole treatment failure in immunocompromised patients: one with a TR34-L98H azole-resistant Aspergillus fumigatus isolate and the other a Rhizomucor-A. fumigatus co-infection. Such patients probably require surveillance by galactomannan antigen detection and quantitative PCRs for A. fumigatus and Mucorales fungi.

Tsavuconazole, an antifungal azole used to treat invasive fungal diseases (IFDs), is approved as a first-line treatment for invasive aspergillosis and can be used as an alternative treatment for mucormycosis (1). We present 2 cases of IFD and isavuconazole treatment failure in acute myeloid leukemia (AML) patients with prolonged neutropenia after hematopoietic stem-cell transplantation (SCT).

Patient 1, a 52-year-old truck driver with AML (diagnosed in 2017), received a haplo-identical SCT (day 0) 4 months after the diagnosis. The patient had incomplete hematologic reconstitution and experienced graft-versushost disease of the digestive tract (day 0 ), which we treated with corticosteroids and ruxolitinib. We gave the patient oral posaconazole (300 $\mathrm{mg} /$ day, starting day 1$)$ for
IFD prophylaxis, as recommended by the 4th European Conference on Infections in Leukaemia (2). On about day 65 , we diagnosed probable invasive aspergillosis according to the criteria of the European Organisation for Research and Treatment of Cancer Mycoses Study Group $(3,4)$; the patient had fever, neutropenia, and a discrete pulmonary lesion on chest computed tomography (CT), and serum samples were repeatedly positive by an in-house $A$. fumigatus quantitative PCR (qPCR) but negative for galactomannan antigen (Figure) $(1,4)$. On the same day, we switched treatment to oral isavuconazole $(200 \mathrm{mg}$ /day). On day 126 , chest CT showed the persistence of pulmonary lesions, and we switched patient treatment to liposomal amphotericin B (5 $\mathrm{mg}$ /day by injection). Thereafter, serum samples became repeatedly positive for galactomannan antigen and $A$. fumigatus DNA. At day 158, we found TR34-L98H azoleresistant A. fumigatus fungus in his bronchial aspirate. The French National Reference Center for Invasive Mycoses and Antifungals (Paris, France) performed MIC testing using European Committee on Antibiotic Susceptibility Testing methods (https://www.pasteur. $\mathrm{fr} / \mathrm{fr} /$ sante-publique/CNR/les-cnr/mycoses-invasivesantifongiques). The following MICs were obtained: amphotericin B $0.25 \mathrm{mg} / \mathrm{L}$ (susceptibility unknown, no breakpoint available), itraconazole $>8 \mathrm{mg} / \mathrm{L}$ (resistant), isavuconazole $4 \mathrm{mg} / \mathrm{L}$ (resistant), and voriconazole $2 \mathrm{mg} / \mathrm{L}$ (susceptible). The patient died 182 days after the SCT.

Patient 2, a 61-year-old businessman with AML (diagnosed in 2013), received his firstallogenic hematopoietic SCT 4 months after the diagnosis. He experienced a relapse 3 years after the first transplantation, and a second allogenic hematopoietic SCT was performed (day 0), which was followed by severe sepsis with Escherichia coli. An excavated nodule was visible on chest CT (day 4), and the A. fumigatus biomarker was repeatedly positive, suggesting probable invasive aspergillosis according to European Organisation for Research and Treatment of Cancer criteria (3). On day 5, treatment with isavuconazole $(200 \mathrm{mg} /$ day orally) was initiated. Graft-versus-host disease of the digestive tract also developed (day 12) in this patient, which

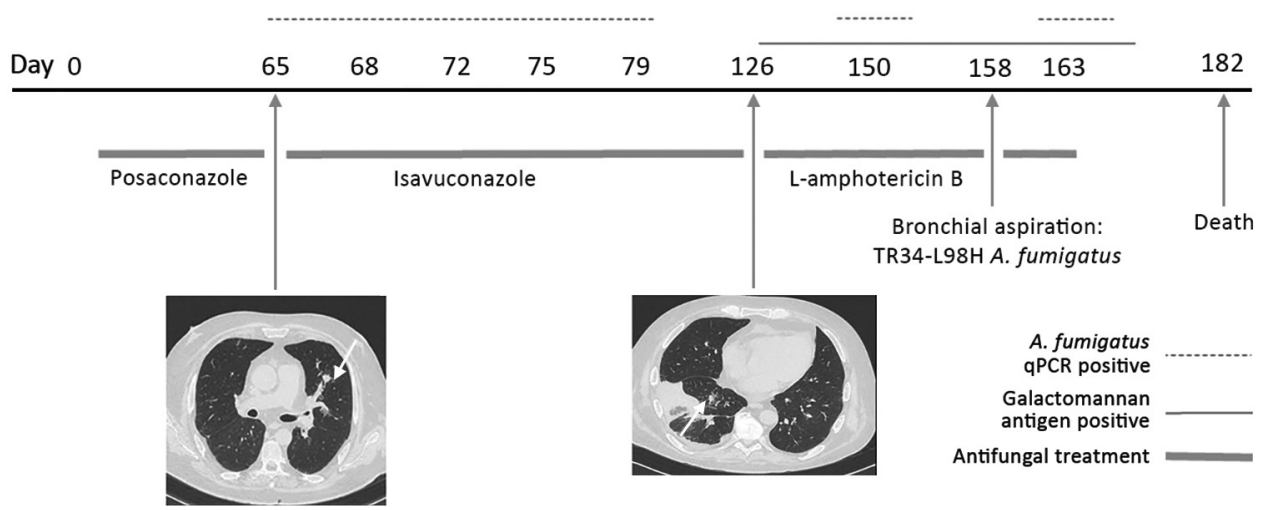

Figure. Evolution of fungal biomarkers, computed tomography chest scans, and antifungal treatments for immunocompromised patient 1 with invasive Aspergillus fumigatus infection, France, 2018. Arrows indicate lesions. qPCR, quantitative PCR. 
we treated with ruxolitinib, tacrolimus, and prednisolone. On day 92, the patient had asthenia, fever, and thoracic pain, and chest CT showed multiple micronodules. On days 95116 , systematic fungal surveillance testing of serum samples showed 1 test positive for galactomannan antigen, 4 positive for A. fumigatus DNA, and 3 positive for Rhizomucor DNA. Two cultured pulmonary samples collected on days 114 and 116 were positive for A. fumigatus. We performed ETESTs (bioMérieux, https://www.biomerieux-diagnostics. com), which indicated the following MICs: amphotericin B $0.023 \mathrm{mg} / \mathrm{L}$ (susceptibility unknown), isavuconazole $0.25 \mathrm{mg} / \mathrm{L}$ (susceptible), and voriconazole $0.38 \mathrm{mg} / \mathrm{L}$ (susceptible). We switched patient treatment to liposomal amphotericin B ( $5 \mathrm{mg} / \mathrm{kg}$ by injection) on day 117 , but the patient died on day 129 .

These 2 cases had in common AML treated by SCT, followed by severe digestive graft-versus-host disease, IFD resistant to isavuconazole diagnosed $>100$ days after SCT, use of combined fungal biomarkers to detect IFD, and death despite rapid prescription of amphotericin B. Severe digestive graft-versus-host disease might have affected the levels of isavuconazole absorbed by the patient because the drug was administered orally in both cases. Intravenous isavuconazole is not recommended for treating IFD and was not available at the treatment facility (University Hospital, Besançon, France). However, these cases suggest that isavuconazole levels should be checked in patients with severe digestive graft-versushost disease. For patient 1, a change in class of antifungal drugs could have been made as early as day 65 , and earlier treatment with amphotericin B could have had a positive effect on his prognosis. The long duration between the initial positive qPCR and galactomannan antigen test result suggests patient 1 might have been infected with multiple $A$. fumigatus isolates, with 1 being resistant. For patient 2, the systematic use of Mucorales qPCR enabled early detection of a mixed Aspergillus-Mucorales fungal infection (5). These types of mixed mold infection were reported to have a prevalence of $25 \%$ in studies including Mucorales qPCR (6). The diagnosis of Rhizomucor infection was based on 3 successive samples being positive by Rhizomucor qPCR. A Mucorales qPCR of the patient's bronchoalveolar lavage fluid (validated assay with satisfying sensitivity) was surprisingly negative (6).

In summary, these cases demonstrate that systematic surveillance is needed for severely immunocompromised patients treated for IFD. Galactomannan antigen detection and qPCRs targeting Aspergillus fumigatus and Mucorales fungi might be the optimal surveillance strategy.

\section{Acknowledgments}

We thank Pamela Albert for her editorial assistance.

\section{About the Author}

Dr. Bellanger is a microbiologist involved in the diagnosis of invasive fungal infections at University Hospital, Besançon, France. Her research focus is on invasive aspergillosis and mucormycosis.

\section{References}

1. Cresemba $^{\circledR}$ (isavuconazium sulfate) [package insert]. Northbrook (IL): Astellas Pharma US, Inc.;2015.

2. Groll AH, Castagnola E, Cesaro S, Dalle JH, Engelhard D, Hope W, et al.; Fourth European Conference on Infections in Leukaemia; Infectious Diseases Working Party of the European Group for Blood Marrow Transplantation; Infectious Diseases Group of the European Organisation for Research and Treatment of Cancer; International Immunocompromised Host Society; European Leukaemia Net. Fourth European Conference on Infections in Leukaemia (ECIL-4): guidelines for diagnosis, prevention, and treatment of invasive fungal diseases in paediatric patients with cancer or allogeneic haemopoietic stem-cell transplantation. Lancet Oncol. 2014;15:e327-40. https://doi.org/10.1016/ S1470-2045(14)70017-8

3. De Pauw B, Walsh TJ, Donnelly JP, Stevens DA, Edwards JE, Calandra T, et al.; European Organization for Research and Treatment of Cancer/Invasive Fungal Infections Cooperative Group; National Institute of Allergy and Infectious Diseases Mycoses Study Group Consensus Group. Revised definitions of invasive fungal disease from the European Organization for Research and Treatment of Cancer/Invasive Fungal Infections Cooperative Group and the National Institute of Allergy and Infectious Diseases Mycoses Study Group (EORTC/MSG) Consensus Group. Clin Infect Dis. 2008;46:1813-21. https://doi.org/10.1086/588660

4. Ullmann AJ, Aguado JM, Arikan-Akdagli S, Denning DW, Groll AH, Lagrou K, et al. Diagnosis and management of Aspergillus diseases: executive summary of the 2017 ESCMIDECMM-ERS guideline. Clin Microbiol Infect. 2018;24 (Suppl 1):e1-38. https://doi.org/10.1016/j.cmi.2018.01.002

5. Millon L, Scherer E, Rocchi S, Bellanger AP. Molecular strategies to diagnose mucormycosis. J Fungi (Basel). 2019;5:24. https://doi.org/10.3390/jof5010024

6. Scherer E, Iriart X, Bellanger AP, Dupont D, Guitard J, Gabriel F, et al. Quantitative PCR (qPCR) detection of Mucorales DNA in bronchoalveolar lavage fluid to diagnose pulmonary mucormycosis. J Clin Microbiol. 2018;56:e0289-18. https://doi.org/10.1128/ JCM.00289-18

Address for correspondence: Anne-Pauline Bellanger, Department of Parasitology-Mycology, Besançon University Hospital Jean Minjoz, 2 Blvd Fleming, 25030 Besançon CEDEX, France; email: apbellanger@chu-besancon.fr 